\title{
INFRATROCHLEAR NEURALGIA: A PROSPECTIVE SERIES OF SEVEN PATIENTS TREATED WITH INFRATROCHLEAR NERVE BLOCKS
}

Rocío-Nur Villar-Quiles ${ }^{1,2}$, Héctor García-Moreno ${ }^{1,3}$, Diego Mayo ${ }^{1,2}$, Álvaro GutiérrezViedma $^{1,2}$, María-Isabel Ramos ${ }^{4}$, Javier Casas-Limón' ${ }^{5}$, María-Luz Cuadrado ${ }^{1,2}$

${ }^{1}$ Department of Neurology, Hospital Clínico San Carlos, Madrid, Spain

2Department of Medicine, School of Medicine, Universidad Complutense de Madrid (UCM), Madrid, Spain

${ }^{3}$ Department of Molecular Neuroscience, University College London (UCL), London, United Kingdom

4Department of Psychiatry, Hospital Clínico San Carlos, Madrid, Spain

${ }^{5}$ Department of Neurology, Hospital Universitario Fundación Alcorcón, Alcorcón, Madrid, Spain

Corresponding author:

Rocío-Nur Villar-Quiles. Department of Neurology, Hospital Clínico San Carlos, C/ Profesor Martín Lagos s/n, 28040 Madrid, Spain.

e-mail: rocionur@gmail.com

\section{ABSTRACT}

Background: Infratrochlear neuralgia is a recently described painful cranial neuropathy that causes pain in the internal angle of the orbit and the medial upper eyelid, the upper bridge of the nose and/or the lacrimal caruncle. We aim to present seven new cases of infratrochlear neuralgia treated with anaesthetic nerve blocks. Methods: Over an 18-month period, we prospective identified seven cases of infratrochlear neuralgia among the patients attending the Headache Unit in a tertiary hospital. Anaesthetic blocks were performed by injecting 0.5 cc of bupivacaine $0.5 \%$ at the emergence of the nerve above the internal canthus.

Results: All patients were women, and the mean age was 49.1 years (standard deviation, 17.9). The pain appeared at the internal angle of the orbit and/or the medial upper eyelid in six cases, and the whole territory of the infratrochlear nerve 
in one case. Six patients had continuous pain and one had episodes lasting 8-24 hours. All patients showed sensory disturbances within the painful area and tenderness upon palpation of the infratrochlear nerve. Nerve blocks resulted in complete and long-lasting relief in four patients and short-lasting relief in the other three patients.

Conclusions: Infratrochlear neuralgia should be considered among the neuralgic causes of orbital and periorbital pain. Anaesthetic blocks may assist clinicians in the diagnosis and may also be an effective therapy.

KEY WORDS: infratrochlear nerve, infratrochlear neuralgia, nerve block, terminal branch neuralgia, trigeminal nerve, trochlear pain 


\section{INTRODUCTION}

Infratrochlear neuralgia is a painful cranial neuropathy recently described as a cause of orbital or periorbital pain (1). It is characterized by pain in the territory supplied by the infratrochlear nerve (i.e. the internal angle of the orbit and the medial upper eyelid, the upper bridge of the nose and/or the lacrimal caruncle) as well as tenderness upon palpation of the emergence of the infratrochlear nerve above the internal canthus.

So far, eight cases of infratrochlear neuralgia have been reported $(1,2)$. All the reported patients had brief paroxysms of pain in one of the three different locations mentioned above. In addition, all of them had local allodynia between attacks. Triggers such as light touch were identified by a minority of patients. Most patients have been treated with oral drugs (gabapentin) with satisfactory response. Nonetheless, anaesthetic blocks proved to be a safe and effective treatment in two of the reported patients $(1,2)$.

We aim to present seven new cases of infratrochlear neuralgia treated with anaesthetic blocks in the Headache Unit of a tertiary hospital and thus contribute to a wider description of this condition and its management.

\section{METHODS}

Between May 2015 and October 2016, seven cases of infratrochlear neuralgia were prospectively identified among the patients attending the Headache Unit in a tertiary referral hospital. We registered demographic data and a detailed history, including their complete medical record and the coexistence of other types of headache. Pain characteristics were carefully evaluated, including the exact location of the pain, the intensity and character of the pain, the temporal pattern, any other associated symptoms, and whether or not there was a precipitating event.

A thorough physical and neurological examination was performed, including inspection and exhaustive sensory examination of the orbital and periorbital areas 
as well as palpation of the trochlear area and the emergence of the supraorbital, supratrochlear, infratrochlear and infraorbital nerves. Eye disorders were excluded by a thorough ophthalmological examination, including funduscopy, optometry, slit lamp examination, intraocular pressure measurements, testing of visual fields, and the Schirmer test. Magnetic resonance imaging (MRI) of the brain and the orbit and routine blood workup including erythrocyte sedimentation rate, C-reactive protein, thyroid tests and immunological screening were obtained in all cases to exclude any other underlying disease.

Superficial anaesthetic blockades of the infratrochlear nerve were performed in all cases. We injected $0.5 \mathrm{cc}$ of bupivacaine $0.5 \%$ with a 30 -gauge needle through the internal angle of the orbit just above the internal canthus, i.e. the emergence point of the infratrochlear nerve (Figure 1). After 5 to 10 minutes, we assessed the topographic distribution of the anaesthetized area (Figure 2). The effectiveness of the anaesthetic block was assessed immediately after the procedure and during follow-up.

The patients gave their oral and written consent to be included in this report.

\section{RESULTS}

The main demographic and clinical characteristics of the patients included in this series are shown in Table 1. All six patients were women. Mean age was 49.1 years (standard deviation [SD] 17.9; median 42; range 35-85), and mean age at onset was 49 years (SD 18; median 42; range 34-85). The time since the onset of the symptoms ranged from 24 hours to 1 year. Six patients had previous migraine and one had ipsilateral epicrania fugax. Yet, the appearance of the new pain was not temporally connected to the prior headaches. One patient started to have the pain immediately after nasal trauma, while two patients related the pain to an episode of trochleitis. Trochleitis was satisfactorily treated by steroid injection near the trochlea in both patients, but they were left with pain confined to the territory of the infratrochlear nerve. Four patients did not have any precipitating event. 
Four patients felt pain on the left side, and three had pain on the right side. Specifically, four patients localized the pain at the internal angle of the orbit and the medial upper eyelid, one patient had pain restricted to the medial upper eyelid, another one complained of pain in the internal angle of the orbit and the last one had pain in the whole territory of the infratrochlear nerve including the internal angle of the orbit, the upper eyelid, the lateral aspect of the nasal bridge and the lacrimal caruncle. Pain location did not change during follow-up. Six patients had a continuous pain, while the remaining patient had a strictly episodic pattern with 12 attacks per week lasting 8-24 hours.

Basal pain was graded as mild (2-3 on a 10-point scale, $n=2)$, moderate (4-6 on a 10point scale, $n=3$ ) or severe (7-8 on a 10-point scale, $n=2)$, with either pressing $(n=4)$ or stabbing $(n=3)$ quality. Superimposed on the background pain, three patients experienced exacerbations of severe or very severe pain (8-10 on a 10-point scale), with pressing $(n=1)$, stabbing $(n=1)$ or electric $(n=1)$ quality. Two of these patients had ultrashort paroxysms lasting up to a few seconds and triggered by touch, while the other patient had transient increases of her background pain lasting 1-2 hours coinciding with psychological stress or horizontal eye movements. None showed autonomic symptoms or other accompaniments. In all cases, simple analgesics or non-steroidal anti-inflammatory drugs had been tried, without success.

All patients had hypersensitivity when the emergence of the infratrochlear nerve was palpated at the superointernal angle of the orbit, just above the internal canthus. Sensory examination of the painful area demonstrated sensory dysfunction in all cases, with hyperalgesia and allodynia $(n=3)$, isolated allodynia $(n=1)$, isolated hyperalgesia $(n=1)$, hyperaesthesia $(n=1)$ or hypoaesthesia $(n=1)$. None of the patients had evidence of trochlear inflammation at the time of assessment. Complete blood workup as well as contrast-enhanced MRI of the brain and orbits were normal in all patients.

All patients underwent an anaesthetic blockade of the infratrochlear nerve, as described above (Figure 1). Five to ten minutes after the procedure, an anesthetic area that matched the complete territory of the nerve was verified (Figure 2). No complications were derived from the intervention. Pain relief was immediately 
obtained in all cases, thus confirming the diagnosis of infratrochlear neuralgia. Three patients had an early recurrence after 48 hours, whereas four patients had a long-lasting effect. Two of the patients with early recurrence had partial improvement ( $>50 \%$ decrease in the frequency and intensity of exacerbations), and none of them was prescribed oral drugs. As for the patients with long-lasting effect, one required a second nerve block one month after the first procedure, but all of them were eventually free of pain during a follow-up ranging from 4 to 16 months.

\section{DISCUSSION}

The infratrochlear nerve is one of the terminal branches of the nasociliary nerve, which stems from the first division (ophthalmic division) of the trigeminal nerve. Within the orbit, the nerve runs along the inferior edge of the superior oblique muscle and exits the orbit in an inferior position relative to the throclea. It has three major branches that provide innervation to three distinct areas: the medial aspect of the upper eyelid and the internal angle of the orbit, the lateral aspect of the bridge of the nose and the lacrimal sac and caruncle (3).

Infratrochlear neuralgia was first described in 2015 and, so far, only eight cases have been communicated $(1,2)$. We provide new evidence for the existence of this condition by reporting seven additional cases. The main features of all patients described to date are shown in Table 2. Former and current reports indicate that infratrochlear neuralgia is a female-predominant disorder, and that it may occur at a wide range of ages. Most cases have been idiopathic, but three patients in the current series had a precipitating event. Two of these patients started to have features of infratrochlear neuralgia after a trochleitis was resolved, and one case occurred after nasal trauma. In the cases preceded by trochleitis, swelling of the trochlea could have damaged the infratrochlear nerve due to its anatomical location $(4,5)$. External trauma could be, as well, a precipitating event, as has been shown in supraorbital neuralgia $(6,7)$. Some of the idiopathic cases might be also related to mechanical factors such as subtle trauma during eye movements or compression of the infratrochlear nerve from other structures along its trajectory within the orbit. 
Most patients with infratrochlear neuralgia localize their pain in the internal angle of the orbit and/or the medial upper eyelid, but the upper nasal bridge and the lacrimal caruncle can also be involved. Unlike the previously reported cases, which presented as paroxysmal pain, most of our patients had a continuous pain. Such heterogeneity in the temporal pattern is similar to that described in other pericranial neuralgias that may manifest as a paroxysmal or continuous pain $(8,9)$. In infratrochlear neuralgia, the pain may acquire a pressing, stabbing or electric quality, and may be of mild, moderate or severe intensity, with occasional exacerbations. In a minority of patients, light touch may trigger or aggravate the pain. On physical examination, patients with infratrochlear neuralgia typically exhibit tenderness upon palpation of the emergence of the nerve at the internal angle of the orbit, just above the internal canthus. In addition, all the reported cases had symptoms and signs of sensory dysfunction within the affected area, such as allodynia, hyperalgesia and hypoaesthesia.

Infratrochlear neuralgia may be mistaken for trochlear pain -either trochleitis or primary trochlear headache- given that both disorders cause pain and tenderness in the internal angle of the orbit $(4,10,11)$. Nonetheless, trochlear pain has a wider distribution, exceeding the territory supplied by the infratrochlear nerve. Moreover, trochlear pain is typically continuous and is exacerbated by vertical eye movements. As stated above, trochlear pain and infratrochlear neuralgia may be somehow related due to the anatomical proximity of the trochlear apparatus and the infratrochlear nerve (5). However, the therapeutic approach should be different. While trochlear pain responds to injections of corticosteroids near the trochlea (11), infratrochlear neuralgia has been treated with antiepileptics or superficial anaesthetic blocks with excellent response $(1,2)$ Although trochlear injection of corticosteroids has not been tried in infratrochlear neuralgia, we have not registered alleviation of the pain from infratrochlear neuralgia upon treatment of trochleitis.

Besides trochlear pain, other disorders can cause pain in the orbital and periorbital region and should be differentiated from infratrochlear neuralgia. That is the case of first-division trigeminal neuralgia (12-14), which affects a wider area and appears as electric shock-like pains commonly provoked by innocuous stimuli. 
Other terminal branch neuropathies such as supraorbital neuralgia $(6,7)$, lacrimal neuralgia $(15,16)$ and infraorbital neuralgia $(17,18)$ may be distinguished by the anatomical distribution of the pain. Trigeminal autonomic cephalalgias may also cause pain in the orbital and periorbital region, but they are typically associated with autonomic features (12,19-21). Migraine may also affect the orbital and periorbital area, but the pain in migraine is normally more diffuse and has typical migrainous accompaniments $(12,22)$. Primary stabbing headache commonly affects the periocular area, but the pain lasts just a few seconds and its location shows great variability even within the same individual $(12,23)$. Idiopathic ophthalmodynia and idiopathic rhinalgia also produce pain in this region $(24,25)$, but the distribution of the pain affects the eye globe or the nose without matching the distribution of any particular nerve. Several disorders of the eye and the orbit $(26,27)$ must be also considered, but they can be appropriately excluded by ophthalmological and radiological exams.

In conclusion, infratrochlear neuralgia should be considered amongst the causes of orbital and periorbital pain and has distinct clinical and exploratory features. Anaesthetic blocks may assist clinicians in the diagnosis and may also be a safe an effective therapy. 


\section{CLINICAL IMPLICATIONS}

- Seven more cases of infratrochlear neuralgia have been reported, expanding the spectrum of the disease.

- All patients were treated with anaesthetic nerve blocks with short or longlasting response, and no complications were derived from the procedure.

- Infratrochlear neuralgia should be considered in the differential diagnosis of orbital and periorbital pain, and it may be treated safely and effectively with anaesthetic blockades of the infratrochlear nerve.

\section{FUNDING}

This research received no specific grant from any funding agency in the public, commercial, or not-for-profit sectors.

\section{CONFLICT OF INTEREST}

None declared.

\section{ACKNOWLEDGEMENTS}

This study was presented in part at the LXVIII Annual Meeting of the Spanish Neurological Society (Valencia, Spain, Nov 15-19, 2016). 


\section{REFERENCES}

1. Pareja JA, Casanova I, Arbex A, et al. Infratrochlear neuralgia. Cephalalgia 2015; 35: 1202-1207.

2. Garcia-Moreno H, Cuadrado ML. Infratrochlear nerve block for a new infratrochlear neuralgia. Cephalalgia 2016; 36: 395-396.

3. Rouvière H, Delmas A. Anatomie humaine: Descriptive, topographique et fonctionnelle. 15 edn. 2002.

4. Yangüela J, Pareja J a, Lopez N, et al. Trochleitis and migraine headache. Neurology 2002; 58: 802-805.

5. Helveston EM, Merriam WW, Ellis FD, et al. The trochlea. A study of the anatomy and physiology. Ophthalmology 1982; 89: 124-133.

6. Sjaastad O, Stolt-Nielsen A, Pareja JA, et al. Supraorbital neuralgia. On the Clinical manifestations and a possible therapeutic approach. Headache 1999; 39: 204-212.

7. Pareja JA, Caminero AB. Supraorbital neuralgia. Curr Pain Headache Rep 2006; 10: 302-305.

8. Gadient PM, Smith JH. The neuralgias: diagnosis and management. Curr Neurol Neurosci Rep 2014; 14: 459.

9. Bajwa ZH, Smith SS, Khawaja SN, et al. Cranial neuralgias. Oral Maxillofac Surg Clin North Am 2016; 28: 351-370.

10. Yangüela J, Sánchez-del-Rio M, Bueno A, et al. Primary trochlear headache: a new cephalgia generated and modulated on the trochlear region. Neurology 2004; 62: 1134-1140.

11. Pareja JA, Sánchez-del-Río M. Primary trochlear headache and other trochlear painful disorders. Curr Pain Headache Rep 2006; 10: 316-320.

12. Headache Classification Committee of the International Headache Society (IHS). The International Classification of Headache Disorders, 3rd edition (beta version). Cephalalgia 2013; 33: 629-808.

13. Haviv Y, Khan J, Zini A, et al. Trigeminal neuralgia (part I): Revisiting the clinical phenotype. Cephalalgia 2016; 36: 730-746.

14. Cruccu G, Finnerup NB, Jensen TS, et al. Trigeminal neuralgia. Neurology 2016; 87: 220-228. 
15. Pareja JA, Cuadrado ML. Lacrimal neuralgia: So far, a missing cranial neuralgia. Cephalalgia 2013; 33: 1198-1202.

16. Cuadrado M-L, Gutiérrez-Viedma Á, Silva-Hernández L, et al. Lacrimal nerve blocks for three new cases of lacrimal neuralgia. Headache 2016. DOI: 10.1111/head.12985.

17. Taylor RW, Samuels HS. Neuralgia involving the infraorbital nerve: report of case. J Oral Surg (Chic) 1952; 10: 248-250.

18. López Mesonero L, Pedraza Hueso MI, Herrero Velázquez S, et al. Infraorbital neuralgia: a diagnostic possibility in patients with zygomatic arch pain. Neurologia 2014; 29: 381-382.

19. Cohen AS, Matharu MS and Goadsby PJ. Short-lasting unilateral neuralgiform headache attacks with conjunctival injection and tearing (SUNCT) or cranial autonomic features (SUNA) - a prospective clinical study of SUNCT and SUNA. Brain 2006; 129: 2746-2760.

20. Tepper DE. Cluster headache. Headache 2015; 55: 757-758.

21. Prakash S, Patell R. Paroxysmal hemicrania: an update. Curr Pain Headache Rep 2014; 18: 407.

22. Yoon MS, Mueller D, Hansen N, et al. Prevalence of facial pain in migraine: A population-based study. Cephalalgia 2010; 30: 92-96.

23. Pareja JA, Ruiz J, de Islas C, et al. Idiopathic stabbing headache (jabs and jolts syndrome). Cephalalgia 1996; 16: 93-96.

24. Pareja JA, Cuadrado ML, Porta-Etessam J, et al. Idiopathic ophthalmodynia and idiopathic rhinalgia: two topographic facial pain syndromes. Headache 2010; 50: 1286-1295.

25. Pareja JA, Montojo T, Guerrero AL, et al. Idiopathic ophthalmodynia and idiopathic rhinalgia: a prospective series of 16 new cases. Headache 2015; 55: $1430-1435$.

26. Ringeisen AL, Harrison AR, Lee MS. Ocular and orbital pain for the headache specialist. Curr Neurol Neurosci Rep 2011; 11: 156-163.

27. Waldman CW, Waldman SD, Waldman RA. Pain of Ocular and Periocular Origin. Med Clin North Am 2013; 97: 293-307. 


\section{FIGURE LEGENDS}

Figure 1. Infratrochlear nerve block: The needle is inserted through the internal angle of the orbit, just above the internal canthus.

Figure 2. Infratrochlear nerve block: Topographic distribution of the anaesthetized area matching the whole skin territory of the lacrimal nerve. 